\title{
Seroconversion of Hepatitis B Vaccine in Young Bangladeshi Children: A Tertiary Centre Experience
}

\author{
Salahuddin Mahmud1 ${ }^{*}$, Jahida Gulshan², Farhana Tasneem³ ${ }^{3}$, Syed Shafi Ahmed1 \\ ${ }^{1}$ Department of Pediatric Gastroenterology, Hepatology \& Nutrition, Bangladesh Institute of Child Health, Dhaka Shishu \\ (Children) Hospital, Dhaka, Bangladesh \\ ${ }^{2}$ Institute of Statistical Research and Training, University of Dhaka, Dhaka, Bangladesh \\ ${ }^{3}$ Department of Pediatrics, BIHS General Hospital, Diabetic Association of Bangladesh, Dhaka, Bangladesh \\ Email: ^drsmbablu@gmail.com
}

How to cite this paper: Mahmud, S., Gulshan, J., Tasneem, F. and Ahmed, S.S. (2021) Seroconversion of Hepatitis B Vaccine in Young Bangladeshi Children: A Tertiary Centre Experience. World Journal of Vaccines, 11, 7-18.

https://doi.org/10.4236/wjv.2021.112002

Received: July 22, 2020

Accepted: February 5, 2021

Published: March 9, 2021

Copyright $\odot 2021$ by author(s) and Scientific Research Publishing Inc. This work is licensed under the Creative Commons Attribution International License (CC BY 4.0).

http://creativecommons.org/licenses/by/4.0/

\begin{abstract}
Background: Hepatitis B virus (HBV) infection is one of the most important global health problems and active immunization is the single most important and effective preventive measure against HBV infection. Several studied show that HBV carrier rate is between $2 \%-7 \%$ in Bangladesh. Bangladesh introduced hepatitis $B$ vaccination in children through Expanded Program on Immunization (EPI) in 2005 that includes 3 doses which starts from six weeks after birth. Currently booster vaccination is not recommended any more. However, many studies on different countries observed a declined level of HBs-antibody over a period of 3 - 6 years that may even reach to non-protective levels. Objective: To evaluate the status of seroconversion and seroprotection along with non-responders of EPI vaccinated children against HBV and to measure their antibody levels in different age groups. Methods: A cross sectional descriptive study was done in the department of Pediatric Gastroenterology, Hepatology \& Nutrition, Dhaka Shishu (Children) Hospital, Dhaka, Bangladesh on 120 cases of EPI vaccinated children enrolled from January-December 2019 while attending the inpatient department without any liver problem. The development of Anti-HBs titre greater than or equal to $10 \mathrm{mIU} / \mathrm{mL}$ is considered as protective immunity and any titre less than 10 $\mathrm{mIU} / \mathrm{mL}$ as non-protective following HBV vaccination. Results: Age of the children was $1-12$ years with mean age of $5.6 \pm 1.7$ years and male: female ratio was 1.1:1. Among the children, $56(46.6 \%)$ were from $1-5$ years age, 36 (30.1\%) children from 6 - 10 years age group and 27 (23.3\%) children from 11 - 12 years age group. Out of 120 children, presence of Anti-HBs protective titre was in 63 (52.5\%) children and non-protective level in 57 (47.5\%) child-
\end{abstract}


ren. Among protective level, $34(60.7 \%)$ children were in 1 - 5 years age group, $18(50.0 \%)$ children in 6 - 10 years age group and $11(39.3 \%)$ children in 11 - 12 years age group. Total 24 (20\%) children were completely non-responder (antibody titre $0.00 \mathrm{mIU} / \mathrm{mL}$ ). Out of 120 mother, 06 (5\%) were HBV positive. Among them 05 (83.33\%) children had Anti-HBs less than $10 \mathrm{mIU} / \mathrm{mL}$. Conclusion: After primary vaccination, a good immune response was detected against hepatitis B virus but it goes below even up to non-protective level with the increase of age. Half of the studied children had non-protective titre after 5 years and one-fifth children totally non-responder after primary hepatitis B vaccination. A booster dose may be recommended after 5 years for optimum seroprotection.

\section{Keywords}

Hepatitis B Virus, Expanded Program on Immunization, Immune Response, Antibody Titre

\section{Introduction}

Hepatitis B virus (HBV) is an enveloped deoxyribonucleic acid (DNA) virus that infects the liver and causes hepatocellular necrosis and inflammation [1]. HBV infection can be either acute or chronic, and may range from asymptomatic infection or mild disease to severe or rarely fulminant hepatitis [2]. Around 2 billion people in the world have evidence of past or present infection with HBV and 257 million are chronic carriers of HBV surface antigen (HBsAg) [1] [2]. Worldwide, it is estimated that around 686,000 people die each year from the complications of chronic hepatitis B virus infection [1] [2] [3] [4].

Age-specific HBsAg seroprevalence varies markedly by geographical region in different parts of the world. An HBsAg prevalence of more than $8 \%$ defines highly endemic areas, a prevalence of $5 \%-7 \%$ defines high intermediate, $2 \%$ $4 \%$ low intermediate, and less than $2 \%$ defines low endemic areas [5]. The highest HBsAg is observed prevalence in the African (6.1\%) and Western Pacific region (6.2\%). Central America, North America and Western Europe are low endemic regions [1] [2] [5]. From Southeast Asia, Bangladesh is in low intermediate endemic zone with the prevalence of $2.9 \%$ [6].

In children, most of the burden of HBV-related disease results from infections acquired in infancy through perinatal or early childhood exposure to HBV [5]. The disease is also transmitted through percutaneous or mucosal exposure to infected blood and various body fluids [1] [2]. The rate of development of chronic HBV infection is inversely related to the age at acquisition of the infection, occurring in approximately $80 \%-90 \%$ infants infected perinatally, $30 \%$ $50 \%$ children were infected before the age of 6 years, and less than $5 \%$ infections occurring in otherwise healthy adults [1] [2] [5]. So, the age of acquisition of $\mathrm{HBV}$ infection is the main determining factor in the clinical expression of the 
acute disease and the development of chronic infection [7] [8].

There is no specific treatment for acute hepatitis B disease. Indication of antiviral therapy (either Tenofovir or Entecavir) for chronic active HBV infection is used to reduce morbidity and mortality due to progressive liver disease [5]. Safe and effective vaccines against hepatitis B have been available since 1982. It is recommended for all children with at least 3 doses of hepatitis $B$ vaccine along with the birth dose [5]. Universal vaccination of hepatitis $B$ has been very effective at preventing infection with the hepatitis $\mathrm{B}$ virus (HBV) and at reducing the development of chronic infection in young children from perinatal or early childhood exposures to HBV [5] [8]. Anti-HBs (HBV surface antibody) is used as a marker of immunity to HBV [5] [7]. Antibody concentration of greater than or equal to $10 \mathrm{mIU} / \mathrm{mL}$ measured 1 to 2 months after administration of the last dose of the primary vaccination series is considered a reliable serological marker of long-term protection against HBV infection [5]. An antibody titre of less than $10 \mathrm{mIU} / \mathrm{mL}$ is considered as non-protective [5] [7] [9]. According to World Health Organization, 185 (95\%) countries of the world have incorporated the Hepatitis B virus vaccine into their neonatal infant immunization schedule [5] [9]. Expanded program on Immunization (EPI) in Bangladesh introduced hepatitis B vaccine since 2005 [6]. The penta-valent, diphtheria-pertussis-tetanus (DPT), hepatitis B (HBV), and Haemophilus influenza type b (Hib) vaccine were incorporated into EPI program while maintaining the immunization schedule at 6, 10 and 14 weeks after birth in 2009 as recommended by the WHO [7] Unfortunately, only 97 (49\%) countries had introduced the recommended birth dose in spite of the availability of vaccine [5]. In 22 (11\%) countries, the hepatitis B birth dose was introduced only for infants born to HBsAg-positive mothers [5]. Bangladesh is one of them. Since perinatal or early postnatal transmission is the most important source of chronic HBV infection globally, all infants (including low birth weight and premature infants) should receive their first dose of hepatitis B vaccine as soon as possible after birth, ideally within 24 hours [5] [7] [9].

Although booster vaccination is not recommended by WHO but many countries over the world observed a declined level of HBs antibody over a period of 3 to 6 years that even reach to non-protective level. On the other side, a few percentage of children completely non-responder even after 3 doses of hepatitis B vaccination. Unfortunately, there is no comprehensive and authentic data regarding the seroprotective rate of hepatitis B vaccine especially following EPI program in Bangladesh. With these backgrounds, in this study we evaluated seroconversion and seroprotection status along with non-responders of EPI vaccinated children against HBV and measured their antibody levels in different age groups.

\section{Materials \& Methods}

\subsection{Study Design}

A cross sectional study was done in the Department of Pediatric Gastroenterol- 
ogy, Hepatology \& Nutrition, Dhaka Shishu (Children) Hospital, Dhaka, Bangladesh from January 2019 to December 2019. A total of 120 consecutive cases of EPI vaccinated children ( 3 doses completed) while attending the inpatient department of our hospital, all aged between 1 to 12 years without any liver problem were enrolled irrespective of their gender after obtaining informed written consent. Children aged less than 1 year or more than 12 years and children who received only 1 or 2 doses of vaccine or any immunosuppressive therapy were excluded from the study. Data was collected on a specially designed questionnaire. Study population was divided into 3 age groups ( 1 - 5 year, 6 - 10 year and 11 - 12 years). Anti-HBs titre in different age, sex and maternal history of infection with $\mathrm{HBV}$ were obtained.

\subsection{Laboratory Tests}

Three $\mathrm{mL}$ of blood sample was taken from anti-cubital vein with full aseptic precaution from each child. Serum was separated from blood samples at collection place. Sample was stored at $-20^{\circ} \mathrm{C}$ temperatures until transported to virology laboratory, Popular Diagnostic Centre (Shamoly branch), a renowned diagnostic center of the country, for proper testing and result. Anti-HBs assay was done by using Chemiluminescent immunoassay (CLIA) technology for the quantitative determination of antibody to hepatitis B surface antigen (Anti-HBs) in human serum or plasma samples.

\subsection{Operational Definitions}

The development of Anti-HBs titre greater than or equal to $10 \mathrm{mIU} / \mathrm{mL}$ was considered as protective immunity (Seroprotection) and any titre less than 10 $\mathrm{mIU} / \mathrm{mL}$ as non-protective following HBV vaccination. When any amount of antibody titre was present in response to vaccine, it was called Seroconversion. On the other side, depending upon the response following vaccination, if antibody titre was greater than $100 \mathrm{mIU} / \mathrm{mL}$ it was called well responder, between $\geq 10-100 \mathrm{mIU} / \mathrm{mL}$ was called responder, 1 to $9 \mathrm{mIU} / \mathrm{mL}$ was partial responder and $0.0 \mathrm{mIU} / \mathrm{mL}$ was called non-responder (Figure 1).

\subsection{Statistical Analysis}

A structured form was used as a data collection instrument. The Statistical Package for Social Science (SPSS) version 24 was used for data analysis.

\section{Results}

\subsection{Basic Features of the Reported Cases}

The age of the children ranged from 1 to 12 years with a mean age of $5.6 \pm 1.7$ years. All the children were classified into one of the three age groups. Among all participants, 56 (46.6\%) children from 1 - 5 year age group, 36 (30.1\%) children from 6 - 10 year age group and 27 (23.3\%) from $11-12$ year age group (Table 1). 


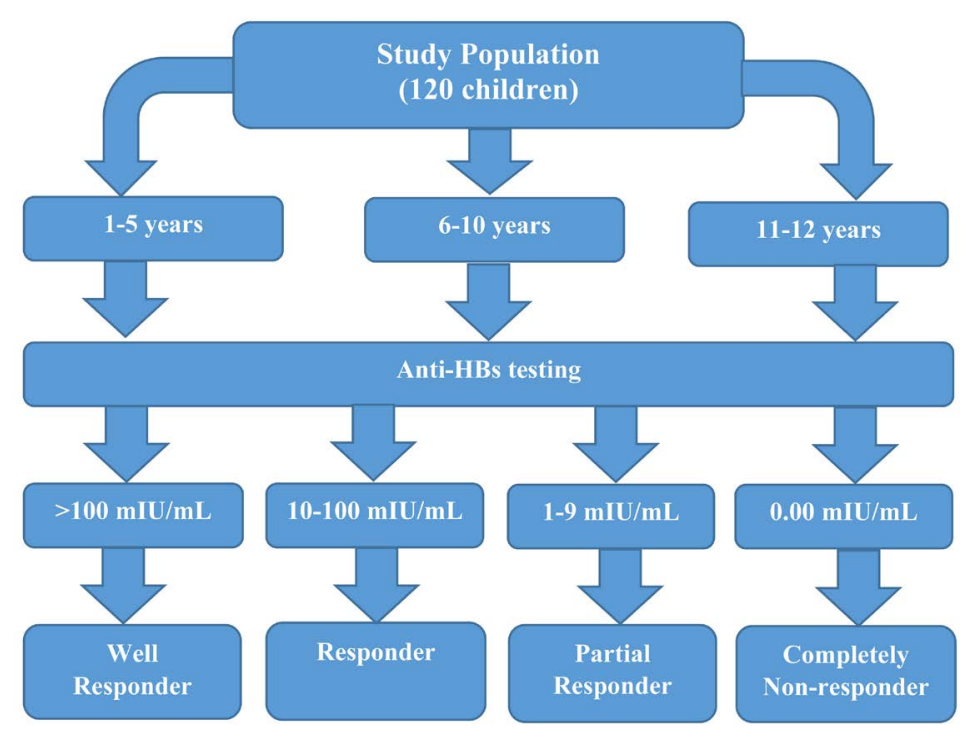

\section{Make conclusions \& further recommendations}

Figure 1. Study flow chart.

Table 1. Age of children.

\begin{tabular}{cc}
\hline Age group (Year) & Frequency (\%) \\
\hline $1-5$ & $56(47 \%)$ \\
$6-10$ & $36(30 \%)$ \\
$11-12$ & $28(23 \%)$ \\
\hline
\end{tabular}

\subsection{Anti-HBs Titre in Different Gender}

Out of 120 children, 63 were male and 57 were female. Male female ratio was 1.1:1 (Table 2). The mean anti-HBs antibody titres among seropositive (anti-HBs $>10 \mathrm{mIU} / \mathrm{mL})$ male $(\mathrm{n}=63)$ and female $(\mathrm{n}=57)$ participants were 159.9 $\mathrm{mIU} / \mathrm{L}$ and $65.9 \mathrm{mIU} / \mathrm{L}$, respectively and there was a significant difference between the anti-HBs of two genders (p-value $<0.05$ ).

\subsection{Seroprotection and Seroconversion of Vaccinated Children against HBV}

The participants were divided into three groups by their antibody titre. The three groups are less than $10 \mathrm{mIU} / \mathrm{mL}, 10-100 \mathrm{mIU} / \mathrm{mL}$ and greater than 100 $\mathrm{mIU} / \mathrm{mL}$ (Figure 2).

Among 120 children, $63(52.5 \%)$ were protective $(\geq 10 \mathrm{mIU} / \mathrm{mL})$ titre and 57 $(47.5 \%)$ were non-protecting titre $(<10 \mathrm{mIU} / \mathrm{mL})$. So, near half of the study population had non-protective titre (Table 3 ).

Among the study population, 21 (17.5\%) children were well responder, 42 (35\%) were responder, 33 (27.5\%) were partial responder and 24 (20\%) were non-responder. So, seroconversion in the studied children was $96(80 \%)$ but seroprotection was 63 (52.5\%) (Figure 3 ). 


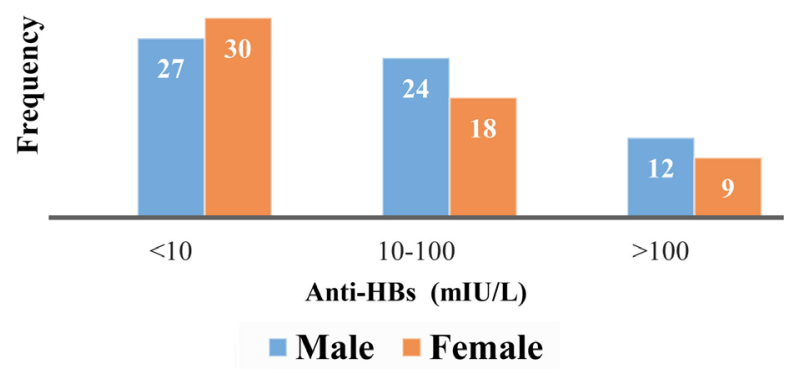

Figure 2. Anti-HBs titre by gender among study population $(\mathrm{n}=120)$.

\section{Responders}

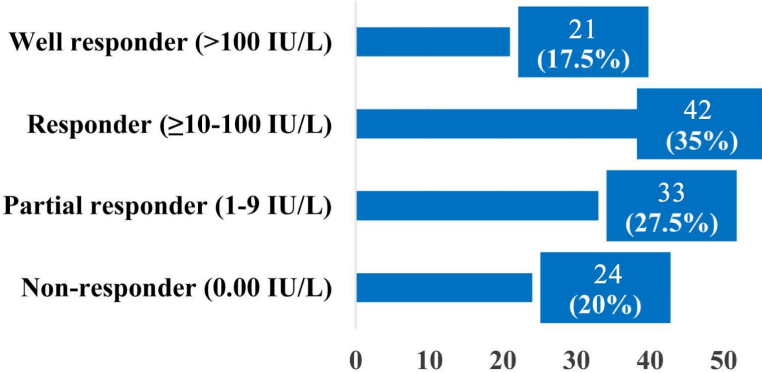

Figure 3. Identification of seroprotection and seroconversion by antibody titre $(n=120)$.

Table 2. Mean seropositive anti-HBs titre by gender among total study population $(\mathrm{n}=$ 120).

\begin{tabular}{cccc}
\hline Gender & Frequency $(\%)$ & Mean anti-HBs titre $(\mathrm{mIU} / \mathrm{mL})$ & P-value \\
\hline Male & $63(52.5)$ & 159.9 & $<0.05$ \\
Female & $57(47.5)$ & 65.9 & \\
\hline
\end{tabular}

Table 3. Anti-HBs positivity among total study population $(\mathrm{n}=120)$.

\begin{tabular}{ccc}
\hline Anti-HBs titre & Frequency & Percentage (\%) \\
\hline$<10(\mathrm{mIU} / \mathrm{mL})$ & 57 & 47.5 \\
$\geq 10(\mathrm{mIU} / \mathrm{mL})$ & 63 & 52.5 \\
\hline
\end{tabular}

\subsection{Anti-HBs Positivity in Different Age Group}

Among study population, $34(60.7 \%)$ in 1 - 5 year age group, $18(50.0 \%)$ in 6 - 10 year age group and $11(39.3 \%)$ in 11 - 12 year age group having protective titre $(\geq 10 \mathrm{mIU} / \mathrm{mL})$. So, protective level of $\mathrm{HBV}$ antibody gradually decreased with increase of age. In the studied children, around $50 \%$ were unprotected $(<10$ $\mathrm{mIU} / \mathrm{mL}$ ) after 5 years and $60.7 \%$ were unprotected after 10 years of age (Figure 4).

\subsection{Completely Non-Responders Following Hepatitis B Vaccination}

Out of 120 children, total 24 (20\%) children was completely non-responder (antibody titre $0.00 \mathrm{mIU} / \mathrm{mL})$. Nine $(16.0 \%)$ children was non-responder in 1 - 5 


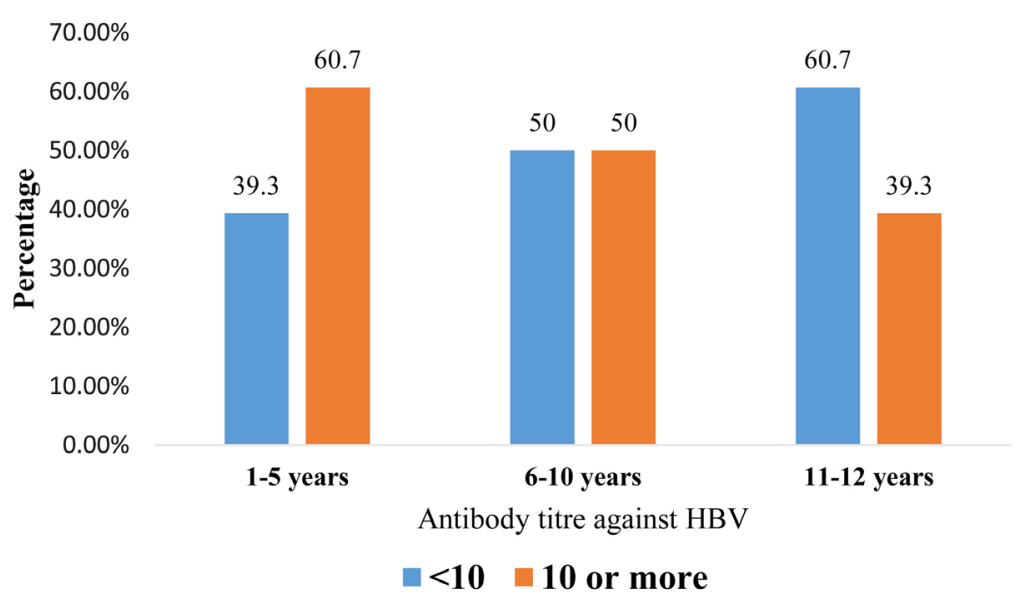

Figure 4. Anti-HBs positivity in different age group $(n=120)$.

year age group, 9 (25.0\%) children in 6 - 10 year age group and $6(21.4 \%)$ children in $11-12$ year age group. There was no significant difference between non-responder age groups (Figure 5).

\subsection{Relationship between H/O Infected Mother and Anti-HBs Titre}

Out of 120 mother, 06 (5\%) were HBV positive. Among them 05 (83.33\%) children had Anti-HBs $<10 \mathrm{mIU} / \mathrm{mL}$. So with the history of HBV positive mother, more chance of appearance of non-protective immunity (Table 4).

\section{Discussion}

Male female ratio of 1.1:1 shows that the infection may occur irrespective of gender. However, mean anti-HBs antibody titre among seropositive (anti-HBs > $10 \mathrm{mIU} / \mathrm{mL}$ ) male and female participants varied significantly $(159.9 \mathrm{mIU} / \mathrm{mL}$ and $65.9 \mathrm{mIU} / \mathrm{mL}$ respectively for male and female. Hossain et al. [7] from Bangladesh and Mazahi et al. [10] from Egypt also reported similar results. In contrast, a study on Iran reported that there were no gender differences in the immunological response to hepatitis B vaccine [11]. T-cell dependence of the immune response may vary in different gender and other than gender, antibody response also depends on age, genetic factors, co-morbidity and the status of immune system of the vaccinee [12].

This study found that nearly half of the study population (47.5\%) had low titre which corresponds to non-protective level. An earlier study from Bangladesh, Hossain et al. [7] observed a similar amount of seroprotection (54\%). Seroprotection of different countries like Taiwan (35.9\%), Saudi Arabia (38.0\%), USA (24.0\%) and Slovakia (48.4\%) also stated the near similar results [13]. According to WHO position paper, children who previously responded to a complete series of hepatitis B vaccination with antibody levels greater than $10 \mathrm{mIU} / \mathrm{mL}$, up to $50 \%$ of vaccinees have low or undetectable concentrations of anti-HBs (anti-HBs loss) between five and 15 years after vaccination [12]. 


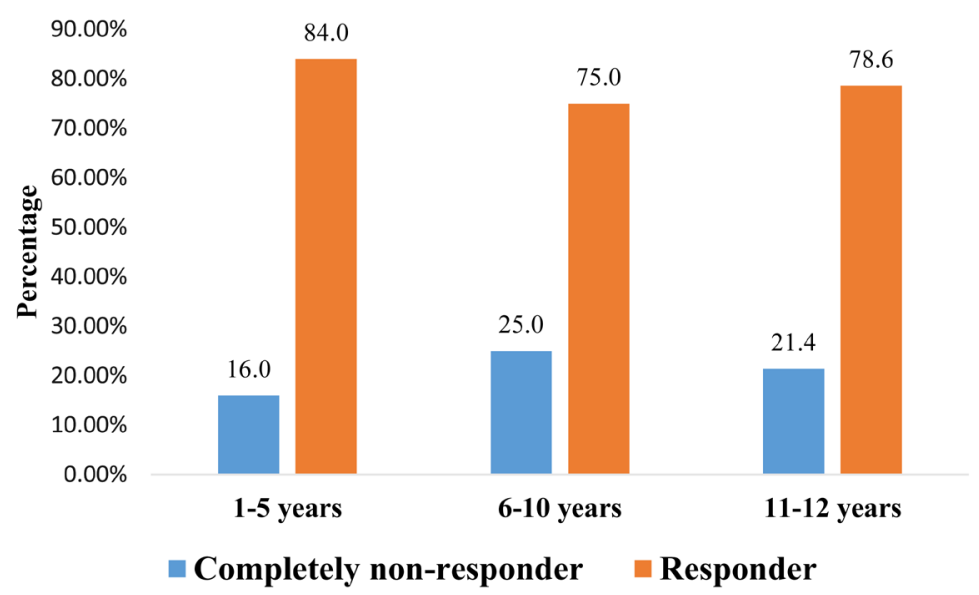

Figure 5. Completely non-responders following Hepatitis B vaccination in different age group $(\mathrm{n}=120)$.

Table 4. Relationship between H/O infected mother and Anti-HBs titre $(\mathrm{n}=120)$.

\begin{tabular}{ccccc}
\hline Infected mother & Total & $\begin{array}{c}<10 \mathrm{mIU} / \mathrm{mL} \\
\text { (Percentage) }\end{array}$ & $\begin{array}{c}\geq 10 \mathrm{mIU} / \mathrm{mL} \\
\text { (Percentage) }\end{array}$ & P value \\
\hline Yes & $06(05)$ & $05(83.33)$ & $01(16.7)$ & 0.07 \\
No & $114(95)$ & $52(45.6)$ & $62(54.4)$ & \\
& $120(100 \%)$ & $57(47.5 \%)$ & $63(52.5 \%)$ &
\end{tabular}

The number of seroconversion (Anti-HBs 80\%) was higher than seroprotection (Anti-HBs 52.5\%) in the studied subject. Other studies from Bangladesh (99.9\% and 92.2\%) [7] [14] and abroad (Brazil-98\%, Srilanka-90.5\% and Ghana-100\%) [15] [16] [17] observed the comparatively better result regarding seroconversion. Anti-HBs antibody is used as a marker of serocoversion or immunity to HBV. A primary 3 doses series induces protective antibody concentrations in $>95 \%$ of healthy infants, children and young adults. Thus, anti-HBs antibody or effective seroconversion is considered reliable serological marker of long term protection against HBV infection [5].

The present study shows that the protective level of HBV antibody gradually decreasing with increase of age (Figure 4). Several studies conducted in different countries for example from Bangladesh [7], South Korea [18], Yemen [19], China [20], Ghana [17] and Palestine [21] observed the similar results. Now the question is, whether the booster vaccination is needed or not. With these query International arena divided into two opinions. WHO stated that, after primary immunization with HBV vaccine, anti-HBs concentrations wane quite rapidly within the first year and more slowly thereafter resulting a higher probability of falling below the $10 \mathrm{mIU} / \mathrm{mL}$. However, anti-HBs concentrations decline to below $10 \mathrm{mIU} / \mathrm{mL}$, immune memory continues to persist over a longer time period. Without this seroprotective level of anti-HBs antibody responded with a rapid rise in titre after a booster dose, indicating immunologic memory and persistence of protection. They also added, protection against HBV infection was 
maintained even though about two third of vaccinees had anti-HBs antibody level $<10 \mathrm{mIU} / \mathrm{mL}$ after 30 years. Thus WHO, as well as advisory groups in Europe and the USA, do not recommend a booster dose in immunocompetent individuals [5] [12]. These statements were supported by several studies from Bangladesh [7], China [22], USA [8] and Iran [23].

However, the recent proclamation from different countries is slightly different saying. They thought that, if the exposed child is suffering with viral illness, immunocompromised disease or drugs, memory cell not adequately activated \& may appear HBsAg on exposure to HBV [18]. On the other hand, it is difficult to know the present ability and status of memory cell of vaccinated individual. A study from South Korea and Japan was found anti-HBs titres in 50\% children were seronegative by 7 years and zero by 13 years. They recommend one booster dose if post vaccination screening is suggestive at the age of 12 - 14 years [18] [24]. A beautiful systematic review and meta-analysis from 12 countries was done by Pakistan. They stated that, risk of having anti-HBs titre $<10 \mathrm{mIU} / \mathrm{mL}$ reduced by $50 \%$ after receiving the booster dose [13]. A recent statement was published in Vaccine journal showing that $25 \%$ of HBV vaccine recipients had an anti-HBs titre $<10 \mathrm{mIU} / \mathrm{mL}$ after 18 years or more from the primary vaccination [25]. They recommended a booster dose of vaccine is safe \& protective for future during early adolescence [25]. Alssamei et al. [19] from Yemen and Hummel et al. [26] from Germany also thought that after 6 - 14 years of infantile HBV vaccination, half of the vaccinees have lost protecting antibody but approximately $90 \%$ of them having immune memory. However, as memory may wane, a booster vaccination is safe and essential for future. Another study from Ghana found that seroprotecting level waned with increase of age, booster doses are therefore recommended after 5 years [17]. Qawasmi et al. from Palestine also suggested a booster dose against HBV during school years [21]. In that perspective, WHO concluded with continued surveillance and long term monitoring of HBV disease of those children who immunized with only three vaccinations in the first year of life. When they become older, a booster dose might become necessary later in life [12].

Among the studied children, total of $24(20 \%)$ children were completely non-responder or showed absence of antibody after vaccination in different age group evidenced by antibody titre $0.00 \mathrm{mIU} / \mathrm{mL}$. Earlier studies also observed complete absence of seroconversion after primary vaccination in few children [7]. More than $80 \%$ of $\mathrm{HBV}$ positive mother had children having anti-HBs 0.00 $\mathrm{mIU} / \mathrm{mL}$. So, history of HBV positive mother, chance of non-protective immunity might be higher. However, with a very small number of such mothers, we should be careful in making generalized comments. On the other side, global coverage of birth dose of HBV vaccine is still low (49\%). Bangladesh is one of these countries. In the absence of the universal birth dose, the transmission of HBV infection from mother to child remains a major source of chronic liver disease when infected children become adults [1] [2]. There are evidences that the risk of infection for infants born to HBsAg-positive mothers may increase 
significantly when the first dose of hepatitis B vaccine is received 7 days after birth compared with those vaccinated within 1 to 3 days after birth [5]. Yue et al. from China recommended that, for partial responders, a booster dose should be given. Without seroconversion or absence of any antibody, revaccination should be considered [20].

\section{Limitations of the Study}

First, the study was a single centre study with a limited sample size. Second, the antibody response was not evaluated following booster vaccination.

\section{Conclusion}

After primary vaccination a good immune response was detected against hepatitis B but it decreased gradually with the increase of age. Half of the studied children had non-protective titre (partial responder) after 5 years and one-fifth children totally non-responder and this puts them at risk of infection.

\section{Recommendations}

A birth dose should be introduced as early as possible for preventing mother to child transmission. Post-vaccination serologic testing is very important to evaluate child immunity against HBV infection. A booster dose may be recommended after 5 years for partial responders having optimum seroprotection in the future. For those who are completely non-responder after primary vaccination, revaccination should be done. However, the findings of this study should be carefully interpreted because of the sample size and non-probabilistic sampling.

\section{Acknowledgements}

The authors are thankful to the reviewers for their thoughtful comments and suggestions that helped us to improve the paper.

\section{Consent for Publication}

Written informed consents were obtained from parents of individual participants. Respected parents of individual participants who were involved in this study agree on the publication of data.

\section{Availability of Data and Materials}

The dataset used in the current study is available from the corresponding author on reasonable request.

\section{Funding}

This research and the publication were completely funded by all authors.

\section{Conflicts of Interest}

The authors declare that there is no conflict of interest to disclose. 


\section{References}

[1] World Health Organization (2017) Guidelines on Hepatitis B and C Testing. WHO, Geneva, 1-204.

[2] World Health Organization (2015) Guidelines for the Prevention, Care and Treatment of Persons with Chronic Hepatitis B Infection. WHO, Geneva, 1-166.

[3] Mahmud, S., Rahman, M., Tasneem, F., Afroz, M., Ahmed, S.S. and Hussain, M. (2017) Surface Antigen (HBsAg) Negative Hepatitis B Virus in Bangladesh: Warrants the Screening for Blood Donors. Bangladesh Journal of Child Health, 41, 181-188. https://doi.org/10.3329/bjch.v41i3.36954

[4] World Health Organization (2019) HBV Key Facts. Geneva.

[5] World Health Organization (2017) Hepatitis B Vaccines: WHO Position Paper. 369-392.

[6] Zaman, M.H., Rahman, A. and Yasmin, M. (2018) Epidemiology of Hepatitis B Virus Infection in Bangladesh: Prevalence among General Population, Risk Groups and Genotype Distribution. Genes, 9, 1-16. https://doi.org/10.3390/genes9110541

[7] Hossain, M.M., Alam, A.N., Siddiqua, M., Siddika, A. and Nessa, A. (2018) Immune Response among the Children to Hepatitis B Vaccination: A Community Based Study in Bangladesh. Bangladesh Medical Research Council Bulletin, 44, 103-108. https://doi.org/10.3329/bmrcb.v44i2.38705

[8] Bruce, M.G., Bruden, D., Hurlburt, D., Zanis, C., Thompson, G., Rea, L., et al. (2016) Antibody Levels and Protection after Hepatitis B Vaccine: Results of a 30-Year Follow-Up Study and Response to a Booster Dose. The Journal of Infectious Diseases, 214, 16-22. https://doi.org/10.1093/infdis/jiv748

[9] WHO Position Paper on Hepatitis B Vaccines. http://www.who.int/wer/2009/wer8440.pdf

[10] Mohamad, M., Mazahi, E.I., Hussein, M., Maksoud, H.M.A., Hady, M.A., Ali, M.A., et al. (2016) Long Term Immunity to Hepatitis B Vaccine among a Sample of Secondary School Students in Damietta. Journal of Pharmacology and Toxicology, 11, 27-32. https://doi.org/10.3923/jpt.2016.27.32

[11] Yazdanpanah, B., Safari, M. and Yazdanpanah, S. (2010) Persistence of HBV Vaccine's Protection and Response to Hepatitis B Booster Immunization in 5- to 7-Year-Old Children in the Kohgiloyeh and Boyerahmad Province, Iran. Hepatitis Monthly, 10, 17-21.

[12] World Health Organization (2011) The Immunological Basis of Immunization Series. Module 22: Hepatitis B. 1-30.

[13] Mahmood, S., Shah, K.U. and Khan, T.M. (2018) Immune Persistence after Infant Hepatitis-B Vaccination: A Systemic Review and Meta-Analysis. Scientific Reports, 8, Article No. 12550. https://doi.org/10.1038/s41598-018-30512-8

[14] Khan, T.M. (2006) Evaluation of Immune Status against Hepatitis B Following Hepatitis B Vaccination under EPI Programme.

[15] Freitas, D.A., Motta, M.S., Mussi-Pinhata, M.M., Jorge, S.M., Yoshida, C.F.T. and Souza, C.B.S. (2002) Immunogenicity of Hepatitis B Vaccine in Preterm and Full-Term Infants Vaccinated within the First Week of Life. Vaccine, 20, 1557-1562. https://doi.org/10.1016/S0264-410X(01)00493-5

[16] Perera, J., Perera, B. and Gamage, S. (2002) Seroconversion after Hepatitis B Vaccination in Healthy Young Adults, and the Effect of a Booster Dose. Ceylon Medical Journal, 47, 6-8. https://doi.org/10.4038/cmj.v47i1.6396

[17] Dassah, S., Sakyi, S.A., Frempong, M.T., Luuse, A., Ephraim, R.K.D., Anto, E.O., et 
al. (2015) Seroconversion of Hepatitis B Vaccine in Young Children in the Kassena Nankana District of Ghana: A Cross Sectional Study. PLOS ONE, 10, e0145209. https://doi.org/10.1371/journal.pone.0145209

[18] Lee, K.H., Shim, K.S., Lim, I.S., Chae, S.A., Yun, S.W., Lee, N.M., et al. (2017) Changes in Hepatitis B Virus Antibody Titres Overtime among Children: A Single Center Study from 2012 to 2015 in an Urban of South Korea. BMC Pediatrics, 17, 1-8. https://doi.org/10.1186/s12887-017-0924-7

[19] Alssamei, F.A.A., Sonboli, N.A., Alkumaim, F.A., Alsayaad, N.S., Ahdal, M.S., Higazi, T.B., et al. (2017) Assessment of Immunization to Hepatitis B Vaccine among Children under 5 Years in Rural Areas of Taiz, Yemen. Hepatitis Research and Treatment, 2017, Article ID: 2131627. https://doi.org/10.1155/2017/2131627

[20] Yue, X., Ge, C., Zhuge, S., He, H., Yang, H., Xu, H., et al. (2017) Changes and Analysis of Anti-HBs Titres after Primary Immunization in 1 to 16 Year Old Chinese Children: A Hospital Based Study. Journal of Viral Hepatitis, 25, 373-380. https://doi.org/10.1111/jvh.12818

[21] Qawasmi, M., Samuh, M., Glebe, D., Gerlich, W.H. and Azzeh, M. (2015) Age-Dependent Decrease of Anti-HBs Titers and Effect of Booster Doses Using 2 Different Vaccines in Palestinian Children Vaccinated in Early Childhood. Human Vaccines \& Immunotherapeutics, 11, 1717-1724. https://doi.org/10.1080/21645515.2015.1041687

[22] Zhao, Y.L., Han, B.H., Zhang, X.J., Pan, L.L., Zhou, H.S., Gao, Z., et al. (2019) Immune Persistence 17 to 20 Years after Primary Vaccination with Recombination Hepatitis B Vaccine (CHO) and the Effect of Booster Dose Vaccination. BMC Infectious Diseases, 19, 482. https://doi.org/10.1186/s12879-019-4134-9

[23] Rezaei, M., Nooripoor, S., Ghorbani, R., Ramezanshams, F., Mamishi, S. and Mahmoudi, S. (2014) Seroprotection after Hepatitis B Vaccination in Children Aged 1 to 15 Years in Central Province of Iran, Semnan. Journal of Preventive Medicine and Hygiene, 55, 1-3.

[24] Norizuki, M., Kitamura, T., Komada, K., Sugiyama, M., Mizokami, M., Xeuatvongsa, A., et al. (2019) Serologic Testing of Randomly Selected Children after Hepatitis B Vaccination: A Cross-Sectional Population-Based Study in Lao People's Democratic Republic. BMC Infectious Diseases, 19, 507. https://doi.org/10.1186/s12879-019-4086-0

[25] Pileggi, C., Papadopoli, R., Bianco, A. and Pavia, M. (2017) Hepatitis B Vaccine and the Need for a Booster Dose after Primary Vaccination. Vaccine, 35, 6302-6307. https://doi.org/10.1016/j.vaccine.2017.09.076

[26] Hummel, I.B., Huber, B., Wenzel, J.J. and Jilg, W. (2016) Markers of Protection in Children and Adolescents Six to Fourteen Years after Primary Hepatitis B Vaccination in Real Life. The Pediatric Infectious Disease Journal, 35, 286-291. https://doi.org/10.1097/INF.0000000000000994 